\title{
Crowd Salience Reduces Aversion to Facially Communicated Psychopathy but Not Narcissism
}

\author{
Alicia L. Macchione ${ }^{1} \cdot$ Mitch Brown $^{2} \cdot$ Donald F. Sacco $^{1}$
}

Received: 22 June 2021 / Revised: 26 December 2021 / Accepted: 27 December 2021 / Published online: 26 January 2022

(c) The Author(s), under exclusive licence to Springer Nature Switzerland AG 2022

\begin{abstract}
Despite the adaptive advantages of social affiliation in humans, the benefits of interpersonal contact are nonetheless bounded. The experience of crowding can emerge from an oversaturation of social affiliation, fostering avoidant behaviors and heightening vigilance toward interpersonal threats. Among these features indicative of threat includes facial structures connoting dark personality traits associated with a proclivity toward exploitative behavior. Despite the potential costs imposed by those exhibiting these features, individuals could nonetheless enjoy coalitional benefits from exploitative humans (i.e., protection). Two studies investigated whether crowding would foster aversion or interest toward facial structures connoting psychopathy and narcissism. Although crowd salience heightened tolerance for psychopathy (Study 1), providing evidence for a bodyguard hypothesis, narcissism was similarly aversive regardless of motivational state (Study 2). We frame results from an evolutionary perspective and provide tentative explanations for discrepant signal values through psychopathy and narcissism that could elicit disparate findings.
\end{abstract}

Keywords Crowding $\cdot$ Face perception $\cdot$ Psychopathy $\cdot$ Narcissism $\cdot$ Personality

The complexity of human sociality is adaptive insofar as it facilitates access to various somatic and reproductive benefits. However, the resulting group living necessitates tradeoffs among individuals, wherein they must manage risks associated with social living to maximize benefits. For example, one cost that emerges through increased physical proximity, which increases the risk of threat, is feeling crowded. Despite this increased proximity becoming easier to manage through specific adaptations for sociality (e.g., reciprocal altruism, behavioral immune system), various environments can nonetheless exacerbate risks of increased proximity through crowding and heighten perceptions of environmental threats (Brown \& Sacco, in press). As the population continues to grow, with a projected population of 9.8 billion people by 2050 (United Nations Department of Economic and Social Affairs, 2017), crowding becomes a prevalent environmental stressor for which humans may not

Alicia L. Macchione

Alicia.Macchione@usm.edu

1 The University of Southern Mississippi, Hattiesburg, MS, USA

2 The University of Arkansas, Fayetteville, AR, USA have much evolutionary preparation due to historical reliance on smaller groups. The prevalence of crowding with a growing population thus presents additional threats insofar as it could increase individuals' likelihood of contact with exploitative individuals capable of inflicting physical harm unto group members.

The increased risk posed by crowded environments is thought to have resulted in the evolution of a self-protection system, wherein selection favored humans with perceptual acuity toward physical cues indicative of physical threats (Neuberg et al., 2011). This resulting threat detection system would facilitate motivations to remove oneself from stimuli regarded as interpersonally threatening to reduce the incursion of potential harm. One potential response to threats in crowded environments may be avoidance of group members who appear hostile. One major source of hostility to which humans could possess acuity includes sensitivity to individuals exhibiting dark personality traits, as previous findings demonstrate that threats to physical safety heighten aversion to facial features heuristically associated with exploitative interpersonal styles (e.g., Brown et al., 2017; Solomon \& Lyons, 2020). This research sought to identify how heightened threat sensitivity from crowd salience can influence 
preferences for facial cues implicating individuals as interpersonal threats based through the Dark Triad.

\section{Effects of Crowding on Threat Detection}

Crowding refers to the psychological distress individuals experience when a given space is unable to accommodate the number of individuals within it, which implicates an environment as threatening due to considerable violations of personal boundaries (Gochman \& Keating, 1980; Stockdale, 1978). Within these crowded environments is a motivation for social withdrawal (Baum \& Valins, 1979) and heightened anxiety (Schaeffer \& Patterson, 1980). Such aversive responses would be functional, given that dense populations present several costs (e.g., increased risk of threat; Jones et al., 2008; Møller et al., 1993). Both chronic and acute awareness of this density has further been found to heighten aversion to interpersonal contact, especially among those whose appearance connotes greater risk of infection (Brown $\&$ Sacco, in press; Brown et al., in press; Maeng \& Tanner, 2013; Maeng et al., 2013; Sng et al., 2017).

Although previous work demonstrates crowd salience facilitates reductions in social engagement in domains related to threat avoidance, it could be possible this aversion to potential threats extends to other domains. One domain could include self-protection from those likely to inflict physical harm. That is, crowding could foster aversion toward individuals presenting physical threats due to similarities between threat detection systems for threat avoidance and self-protection (i.e., Neuberg et al., 2011). Such experiences could generalize their aversion to cues diagnostic of interpersonal dominance that are frequently avoided among those in environments with chronically heightened concerns of interpersonal violence (Borras-Guevara et al., 2017).

\section{Facial Cues to Interpersonal Threats}

Human faces are robust social stimuli from which one can infer myriad information. Such information could include personality through specific structures, with individuals having above-chance accuracy in inferring personality through these structures through these heuristic cues (Little \& Perrett, 2007; Parkinson, 2005; Sacco et al., 2018). Within the personality traits that could ostensibly be inferred through facial structure, or at least a behavioral repertoire diagnostic of such, includes the Dark Triad (Alper et al., 2021; Holtzman, 2011; but see Shiramizu et al., 2019). This Dark Triad is a cluster of inter-related personality traits implicated in fostering relatively antisocial interpersonal styles toward group members, conceptualized through psychopathy, narcissism, and Machiavellianism (Paulhus \& Williams, 2002). Individuals exhibiting higher levels of these characteristics are particularly dominant and aggressive (Jones \& Paulhus, 2011), with recent theoretical models suggesting their emergence within a given ecology being to facilitate the exploitation of group members to enhance one's own inclusive fitness (Glenn et al., 2011).

The possibility of such exploitation could have resulted in an evolutionary arms race with perceptual acuity toward facial features indicative of these traits, namely through features indicative of dominance (Holtzman, 2011). Although recent findings indicate a degree of limited acuity toward the specific personality traits connoted through stimuli generated from faces of individuals exhibiting high levels of Dark Triad traits (e.g., Alper et al., 2021; Shiramizu et al., 2019), a signal value of dominance and exploitativeness may nonetheless be apparent through these structures due consistent evidence suggesting a general aversion toward these facial structures across multiple trials (e.g., Brown et al., 2017; Solomon \& Lyons, 2020).

Recent findings have further begun identifying how various motivational states functionally predict preferences for faces connoting the social affordances of Dark Triad traits. Women motivated to acquire short-term mates heighten their preference for narcissistic facial structures to identify mates most interested in these sexual encounters (Marcinkowska et al., 2015). Men further identify the value of psychopathy in selecting coalition members when they believe the world is dangerous (Brown et al., 2017), akin to women's heightened preferences for dominant facial and bodily features in the service of identifying effective protectors (Sacco et al., 2015; Snyder et al., 2011). Nonetheless, such preferences may merely represent a tolerance for Dark Triad traits when they may afford some potential benefit in a given context. Psychopathic and narcissistic facial structures are particularly aversive, especially among women concerned about physical safety, given the potential costs they could inflict upon perceivers (Brown et al., 2017; Medlin et al., 2020; Solomon \& Lyons, 2020). People are by definition close to one another in crowded spaces and thus, Dark Triad traits could be especially harmful in such spaces. Such perceptions could foster considerable motivation for individuals to avoid them when feeling crowded due to increased concerns about physical threats inherent when personal boundaries are violated (Neuberg et al., 2011; Schaeffer \& Patterson, 1980).

\section{Current Research}

The current program of research sought to determine how the activation of a threat detection system through crowd salience shifts preferences for exploitative facial structures, whether through heightening or reducing aversion. We specifically considered two Dark Triad personality traits that appeared most predictive of direct interpersonal threat (Rogoza \& 
Cieciuch, 2020), namely psychopathy (Study 1) and narcissism (Study 2). For both studies, we tested competing hypotheses: the threat management and bodyguard hypotheses. For the former, given the aggressive and exploitative tendencies of Dark Triad individuals, it could be that crowd salience heightens aversion to facial structures connoting these traits (see Solomon \& Lyons, 2020). However, with the latter hypothesis, given that crowds may function as a protective barrier against exploitation, it could be that crowd salience actually leads to greater tolerance for these Dark Triad traits (Brown et al., 2017). For study materials and data: https://osf. io/mxqe4/?view_only=7f3ca7ebf00b4d67a2c8117ee849a5ee

\section{Study 1}

The first study considered the effects of crowding on an individuals' preferences for facial structures connoting psychopathy, a trait commonly associated with high impulsivity and low empathy (Paulhus \& Williams, 2002). Previous findings have indicated such structures are particularly aversive and perceived as dangerous (e.g., Solomon \& Lyons, 2020). The costs of psychopathy could be increased within densely populated environments, as increased proximity could increase the likelihood of contact with exploitative individuals. Given the decrease in social behaviors and anxiety that emerge in the service of threat mitigation within crowded environments that could limit contact with exploitative individuals (Baum \& Valins, 1979; Schaeffer \& Peterson, 1980; Elleman et al., 2020), it could be that crowd salience would heighten aversion to facially communicated psychopathy.

Conversely, interactions with individuals high in psychopathy could present several benefits within crowded environments. Considering the bodyguard hypothesis, perceivers could recruit coalition members in densely populated environments to increase the likelihood of receiving protection from those who could have otherwise been prone to exploit a perceiver. This suggests crowd salience may actually heighten tolerance to facially communicated psychopathy. We tested these competing hypotheses in Study 1.

\section{Method}

Participants We recruited 575 undergraduate students from a large university in Southeastern U.S. in exchange for course credit. We ultimately excluded 31 participants from final analyses for not completing the study in its entirety, leading to a final sample of 544 (429 women, 114 men, 1 identifying as Other; $M_{\mathrm{Age}}=20.37$ years, $S D=4.28$ years; $59 \%$ White). We did not conduct an a priori power analysis but used the end of the semester as the stop rule for data collection. A sensitivity analysis using $\mathrm{G}^{*}$ Power (Faul et al., 2007) for a $2 \times 2$ ANOVA indicated we were adequately powered to detect small effects (Cohen's $f=0.05$, $1-\beta=0.80)$.

\section{Materials and Procedures}

Crowding Prime Consenting participants were initially assigned randomly to one of two immersive scenarios that they were asked to imagine themselves in them. One scenario presented a crowded public space and the other a non-crowded space to serve as a control prime (Maeng et al., 2013). Participants wrote about how they would feel if they were in these situations. After the writing task, participants completed a single-item measuring general affect $(-3=$ Extremely Negative $3=$ Extremely Positive $)$, followed by a 6 -item manipulation check to assess specific feelings of being crowded (e.g., anxious, tense, overwhelmed; $1=N o t$ at All; $7=$ Very Much; $\alpha=0.85$ ).

Psychopathy Faces Participants then viewed 40 pairs of faces manipulated to connote high and low levels of psychopathy (Brown et al., 2017). Faces were generated by blending 20 unique male identities and 20 unique female identities with prototypical faces connoting high and low levels of psychopathy (Holtzman, 2011), using Morpheus software to ensure faces were a $50 \% / 50 \%$ combination of each face (see Brown \& Sacco, 2016). This resulted in each unique identity having a high- and low-psychopathy version presented to participants. Each unique identity was presented to participants in a randomized and counterbalanced order to prevent order and stimulus effects, with participants being tasked to indicate with whom in each face pair presented they would prefer to interact.

Participants indicating a preference for a low-psychopathy face was coded as a 1, with a preference for high-psychopathy a 0 . We calculated participants' preference as a proportion of the number of times they selected the low-psychopathy face relative to the number of trials for each sex separately, resulting in a within-subjects factor of Target Sex. However, for the sake of easier interpretation, we transformed these variables to where a higher score would reflect a preference for high-psychopathy, whereas a lower score would reflect a preference for low-psychopathy (i.e., psychopathy aversion). Figure 1 presents sample stimuli.

\section{Results}

Preliminary Analyses As manipulation checks, we conducted a pair of independent samples t-tests to identify conditional effects on affect and feelings of crowding. Crowding-primed participants reported more negative affect $(M=-0.09, S D=1.49)$ than control-primed participants 
Fig. 1 Examples of male and female targets connoting high (left) and lowlevels of psychopathy (right)

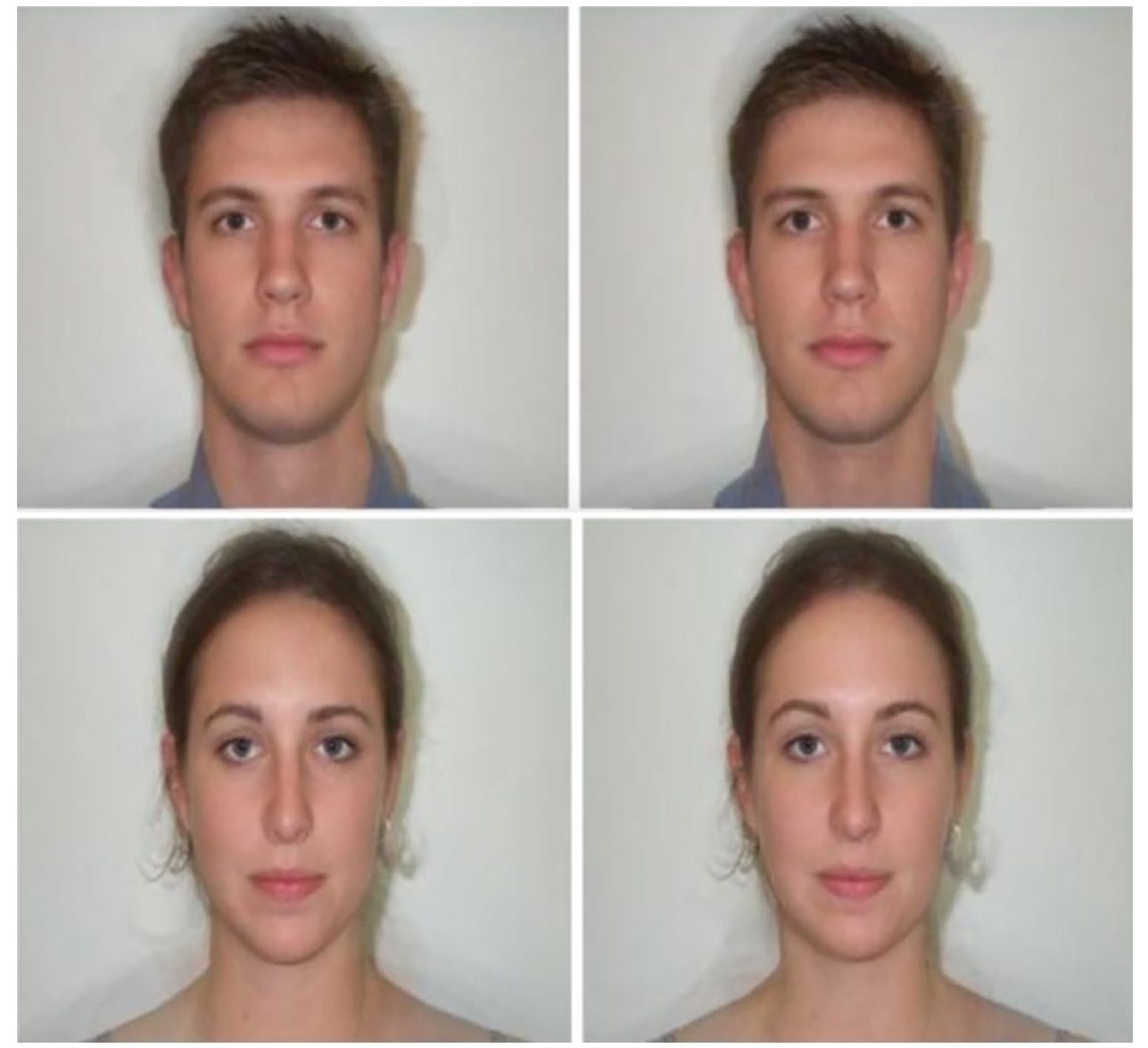

$(M=0.73, S D=1.33), t(542)=-6.73, p<0.001, d=0.57$, 95\% CI [-1.05, -0.58]. Crowding-primed participants indeed reported feeling more crowded $(M=5.51, S D=1.75)$ than did control-primed participants $(M=1.93, S D=1.43)$, $t(531.11)=26.15, p<0.001, d=2.23,95 \%$ CI $[3.31,3.84]$.

Next, we conducted a one samples $t$-tests to identify general preferences towards psychopathy (with a test value of 0.5). Participants reported an overall aversion toward high-psychopathy faces in the both the crowding condition $(M=0.46, S D=0.10), t(279)=-7.44, p<0.001, d=0.44$, $95 \%$ CI $[-0.05,-0.03]$, and the control condition $(M=0.44$, $S D=0.09), t(262)=-10.38, p<0.001, d=0.64,95 \%$ CI $[-0.07,-0.05]$.

Primary Analyses We conducted a 2 (Condition: Crowding vs. Control) $\times 2$ (Target Sex: Male vs. Female) mixed-model ANOVA with repeated factors over the latter factor. A Target Sex main effect indicated a greater aversion toward high psychopathy in female faces $(M=0.44, S D=0.12)$ than male psychopathic faces $(M=0.46, S D=0.14), F(1,541)=8.71$, $p=0.003, \eta_{p}{ }^{2}=0.016,95 \%$ CI [0.01, 0.04]. An additional main effect of Condition indicated crowding-primed participants reported less aversion toward high-psychopathy faces $(M=0.46, S D=0.10)$ than control-primed participants $(M=0.44, S D=0.09)$, albeit still reporting a preference for low-psychopathy faces, $F(1,541)=4.04, p=0.045$, $\eta_{p}{ }^{2}=0.007,95 \%$ CI $[0.00,0.03]$. The interaction was not significant, $F(1,541)=0.20, p=0.653, \eta_{p}{ }^{2}=0.007 .{ }^{1}$ See Table 1 for a summary of primary analyses.

\section{Discussion}

Providing tentative support for the bodyguard hypothesis, crowd salience reduced aversion to high-psychopathy faces. This finding appears to reflect strategic preferences within crowded environments to reduce one's likelihood of incurring harm given the potential danger they may impose. Selecting individuals appearing exploitative could facilitate the formation of coalitional alliances within an ecology that is already highly stressful from proximity violations, reducing the risk of additional interpersonal threats with a "personal protector" (Brown et al., 2017; Snyder et al., 2011). Forming such alliances could further reduce the likelihood of targets viewing perceivers as exploitable from their shared coalitional status despite the potentially greater risk of incurring the cost of exploitation. Nonetheless, this relative tolerance for psychopathy among crowding-primed 
Fig. 2 Examples of male and female targets connoting high (left) and lowlevels of narcissism (right)
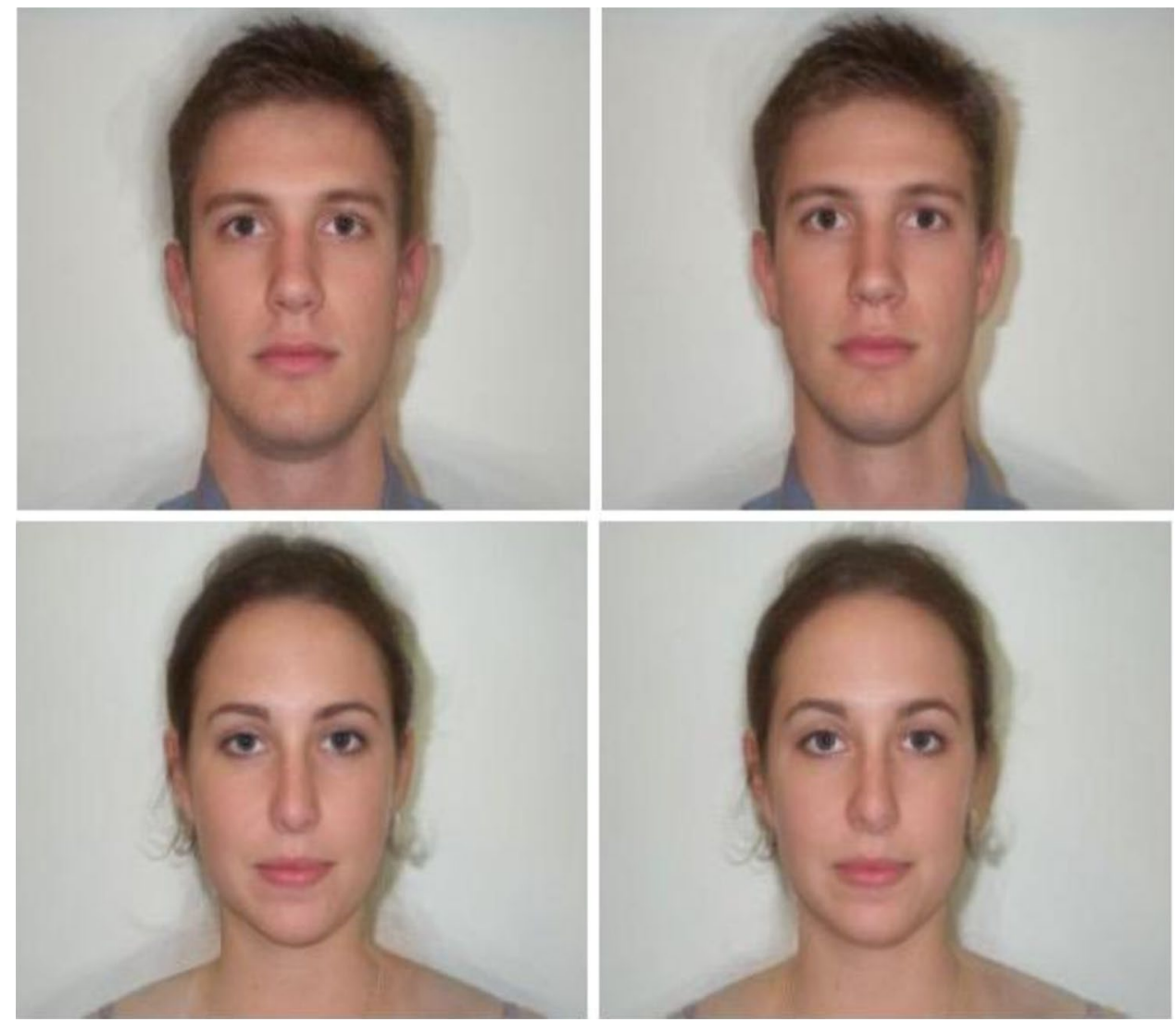

participants is ultimately a reduced aversion, which could reflect considerable salience for the interpersonal costs of psychopathy with crowds merely increasing the salience of a potential benefit.

The potential salience of the benefit from selecting highpsychopathy targets could have led individuals to be willing to prevent exploitation from others by allying themselves with a potential threat. Such an alliance could be specific to psychopathy but not to other facets of the Dark Triad, given that psychopathy is not as typified by a selfish nature that could lead them to see more value in cooperative alliances. Study 2 sought to determine how crowd salience could shift preferences for faces connoting high and low levels of narcissism.

\section{Study 2}

Study 2 sought to determine potential boundary conditions through preferences for narcissism, a trait that may present its own interpersonal costs. Narcissism is a trait commonly associated with heightened grandiosity and entitlement (Paulhus \& Williams, 2002), which is often predictive of aggressive interpersonal strategies following perceived slights (Twenge \& Campbell, 2003).
Narcissism is itself a dominant personality trait, which is further inferred through facial structures connoting dominance in both male and female faces (Holtzman, 2011; Shiramizu et al., 2019). Recent findings further indicate the dominance facets of narcissism are further associated with physical formidability, which could implicate narcissistic individuals as having greater potential for physical exploitation (or coalitional value) similarly to psychopathy (von Borrell et al., 2019). Further, research has found that sociosexually restricted women are particularly averse to narcissistic faces, which could reflect potential wariness to the long-term costs despite the potential benefits (Lyons \& Simeonov, 2016). We tested the same competing hypotheses as in Study 2, albeit now considering narcissism.

\section{Method}

Participants We recruited 646 undergraduate students from a large university in Southeastern U.S. in exchange for course credit. We excluded 37 participants from final analyses for providing incomplete data for a final sample of 609 (525 women, 84 men; $M_{\text {Age }}=21.34$ years, $S D=5.89$ years; $60 \%$ White). A sensitivity analysis for the requisite $2 \times 2$ ANOVA indicated we were adequately powered to detect 
small effects (Cohen's $f=0.05,1-\beta=0.80$ ). We employed the same stop rule for data collection as Study 1 by collecting for the entirety of the semester.

Materials and Procedure Consenting participants engaged in the same priming procedure as Study 1 that included the same manipulation check items for affect and feelings of crowding $(\alpha=0.84)$. This was followed by the same face preference task, albeit with faces manipulated to connote high and low levels of narcissism employing the same manipulation techniques, unique identities, and prototypes as psychopathy (Medlin et al., 2020). Figure 2 presents sample stimuli. We calculated preferences for high narcissism by summing the number of times participants selected the high narcissism faces and created a proportion from the number of trials, separately for male and female faces for 20 trials for each sex to create the same type of within-subjects factor for Target Sex as Study 1.

\section{Results}

Preliminary Analyses We first employed two independent samples t-tests for our manipulation check items. Crowdingprimed participants reported more negative affect $(M=0.03$, $S D=1.43)$ than control-primed participants $(M=0.82$, $S D=1.30), t(607)=-7.01, p<0.001, d=0.58,95 \%$ CI $[-1.01$, -0.57]. Further, crowding-primed participants reported feeling more crowded $(M=5.71, S D=1.70)$ than controlprimed participants $(M=1.92, S D=1.37), t(597.05)=30.49$, $p<0.001, d=2.45,95 \%$ CI $[3.54,4.03]$.

Next, we conducted a one samples $t$-tests to identify general preferences towards narcissism in both conditions (with a test value of 0.5). Participants reported an overall aversion toward high-narcissism faces in the both the crowding condition $(M=0.44, S D=0.12, t(316)=-8.09, p<0.001$, $d=-0.45,95 \%$ CI $[-0.07,-0.04]$ and the control condition, $(M=0.44, S D=0.11), t(291)=-8.81, p<0.001, d=-0.52$, $95 \%$ CI $[-0.07,-0.04]$.

Primary Analyses We conducted a 2 (Condition: Crowding vs. Control) $\times 2$ (Target Sex: Male vs. Female) mixed-model ANOVA with repeated factors over the latter factor. A Target Sex main effect indicated greater aversion toward high narcissism in male faces $(M=0.40, S D=0.17)$ than female narcissistic faces $(M=0.48, S D=0.13), F(1,607)=114$, $p<0.001, \eta_{p}{ }^{2}=0.159,95 \%$ CI $[0.11,0.21]$. The main effect of Condition was not significant, $F(1,607)=0.127, p=0.72$, $\eta_{p}{ }^{2}<0.001$. Further, the interaction was not significant, $F(1$, $607)=0.20, p=0.65, \eta_{p}{ }^{2}<0.001$. A follow-up equivalence test, with $\mathrm{t}$ test statistic of 0.356 and $95 \%$ confidence interval of -0.01 to 0.02 , suggested that the difference between narcissistic preferences between the crowding-primed and control-primed participants was equivalent to 0 , which indicates that the effect of crowding on preferences was essentially zero (Lakens et al., 2018). See Table 1 for a summary of analyses.

\section{Discussion}

Study 2 was consonant with previous research indicating an overall aversion to narcissistic facial structures (Medlin et al., 2020). This aversion could suggest an awareness of narcissism presenting few benefits in the contexts provided in this research unlike the potential utility seen for psychopathy in crowds. Although narcissists present an attractive veneer upon initial meeting (Holtzman \& Strube, 2012), this attractiveness dissipates through continued interaction (Campbell et al., 2005; Paulhus, 1998). It could be possible the salience of a crowd led individuals to orient their interpersonal style toward long-term planning (Sng et al., 2017). Much like with other facial structures diagnostic of interpersonal dominance (Brown \& Sacco, 2017), the longterm costs of narcissism becoming more salient without participants recognizing any possible benefits to the trait in comparison (Marcinkowska et al., 2015).

\section{General Discussion}

Results across both studies provide mixed support for our competing predictions. Although we found evidence for a bodyguard hypothesis for facial structures connoting psychopathy when crowds are salient, no effects of crowding emerged for narcissism. This discrepancy could represent an unexpected nuance to these interrelated traits and may highlight different adaptive bases for their emergence in stressful environments. Whereas individuals could recognize psychopathy as possessing a protective function to reduce the likelihood of exploitation through sustained interpersonal contact (Brown et al., 2017), narcissism's particularly salient longterm costs could preclude them from being seen as capable of enhancing group living in an environment that necessarily

Table 1 Summary of primary findings in Study 1 (Psychopathy) and Study 2 (Narcissism)

\begin{tabular}{llll}
\hline & $F$ & $p$ & $\eta_{p}{ }^{2}$ \\
\hline Study 1 & $F(1,541)$ & & \\
Target Sex & 8.71 & .003 & .016 \\
Crowding & 4.04 & .045 & .007 \\
Interaction & .20 & .653 & .007 \\
Study 2 & $F(1,607)$ & & .159 \\
Target Sex & 114 & .001 & .000 \\
Crowding & .127 & .72 & .000 \\
Interaction & .20 & .65 & \\
\hline
\end{tabular}


fosters slower life history strategies (Marcinkowska et al., 2015; Sng et al., 2017). This distinction between different interpersonal costs for these traits could suggest a need to understand narcissism and psychopathy separately to some degree despite recent research suggesting Dark Triad traits may better be modeled as a single construct (Bertl et al., 2017). That is, the interpersonal function of these personalities seems to produce a level of distinctiveness that warrants research that takes into consideration the facets of Dark Triad traits.

Interestingly, as articulated in footnotes, men and women did not differ in their preferences. One might predict stronger aversion for women, given the asymmetry in physical size that creates a physical disadvantage for women in conflict (Brown et al., 2017; Sell et al., 2012). However, close proximity of humans within a crowded environment could have potentially muted any sex differences in responses. Nonetheless, participants reported differing levels of aversion to psychopathy and narcissism in male and female faces. Contrary to previous findings (Brown et al., 2017), high psychopathy in female faces was slightly more aversive than in male faces, which could reflect an aversion to dominant facial structures not typical of female faces, although this discrepancy necessitates future research. Study 2 saw greater aversion toward high narcissism in male faces than in female faces, a finding that would be more expected, given the greater costs of exploitation imposed by dominant men compared to women. These findings are nonetheless subsumed in the overall aversion to psychopathy and narcissism exhibited by participants across both studies, which could suggest the inconsistencies across male and female faces could be more subject to situational moderators yet to be identified.

\section{Future Directions and Limitations}

Several future directions would be advantageous to clarify some of the nuance in these findings. Despite the utility of the preference task employed in these studies in understanding basic perceptions of these facial structures (i.e., two alternative face choices), such paradigms may lack a degree of ecological validity that could undermine their ability to detect actual preferences for structures connoting personality (Jaeger et al., 2021; Jones \& Jaeger, 2019). This is especially apparent when considering the rather hypothetical nature of these interactions. Future studies could present these faces separately for participants to indicate the degree to which they would be interested in interacting with targets in a crowded (or uncrowded) environment. To reduce concerns of hypotheticality, it would be further advantageous to indicate to participants that they would eventually interact with targets pictured (Montoya \& Insko, 2008). Participants could be shown different targets, one manipulated to be high in psychopathy and one low (but different identities) and told that the two targets are waiting in separate cubicles and allow participants to select the target they would prefer to work with on a task.

Although out findings found a consistent aversion toward facial structures connoting psychopathy, recent work indicates that psychopathy is not as accurately inferred in female faces as it is in male faces (Shiramizu et al., 2019). This potential limitation may suggest that the distinction of psychopathy may be specific to male faces, with the trait being inferred in a different capacity in female faces. Nonetheless, psychopathy in female faces was regarded as particularly aversive in this research in a manner that parallels perceptions of male faces (see Brown et al., 2017), suggesting a similar signal value of interpersonal dominance despite not both being regarded as psychopathy. Future research would benefit from specifically identifying the linguistic basis of the aversion to psychopathy in female faces to determine the similar aversion to these structures. Furthermore, it remains imperative to address recent concerns about the potential veracity of these signal values in facial structures from admittedly limited stimulus sets (DeBruine, 2020; Shiramizu et al., 2019). Future work would benefit from using more extensive stimulus sets to determine whether the preferences identified in these studies are rooted in a generalizable kernel of truth in personality inferences or if these stimuli facilitate an inference for structures typically regarded as dominant.

Although crowding seemed to affect interpersonal preferences psychopathy, it is unclear what the exact motivation for this aversion is. Crowding renders many threats salient, thereby heightening avoidance responses to aversive stimuli that extends into unique threat management responses (e.g., Baum \& Valins, 1979; Brown et al., in press). However, it remains unclear what the specific motivational system activated in response to crowd stimuli. Future research would benefit from assessing which specific threat management responses specifically modulate preferences for facial structures. Understanding which system is activated could further inspire work investigating whether the tolerance for psychopathy in Study 1 could be rooted in shifts in the salience of social opportunities afforded through psychopathy that would compete with ostensibly salient threat affordances (see Brown, 2021).

It is further important to note that Study 1 was conducted prior to the start of the COVID-19 pandemic, whereas Study 2 occurred during the pandemic prior to the deployment of vaccines. It could be possible that the pandemic fostered especially avoidant behaviors among participants regardless of whether or not a crowd is present. Indeed, recent findings suggest a general downregulation for interpersonal contact emerged across a variety of domains during the pandemic (Gul et al., 2021). It could be possible that the overall aversion to interpersonal contact during the pandemic ultimately muted any possibility of identifying the tradeoffs in 
narcissism preferences, even when the primed environment appears particularly pathogenic (Brown \& Sacco, in press). Future research would benefit from specifically considering the effects of Study 2 in an environment without a salient threat related to interpersonal contact.

Finally, the current study's findings should be interpreted cautiously, given the relatively small effect sizes. Future work would benefit from conducting direct replications of these findings to determine their consistency. This interest in direct replication would additionally provide the opportunity to pre-register study predictions.

\section{Conclusion}

The centrality of interpersonal contact for human survival necessitates consideration of whether group members would be likely to exploit others and if individuals would be willing to incur the costs of affiliation to increase security within a stressful ecology. These studies provide tentative evidence for how crowd salience could shape preferences for facial structures connoting dark personality traits in the service of reducing one's risk of harm. Although tentative evidence suggests a tolerance for psychopathy in crowds, ambiguity in these results nonetheless remain that necessitate future work to identify how functional preferences are shaped by such ecological stressors.

Authors' Contributions All authors contributed to the study conception and design. Material preparation and data collection were performed by Alicia Macchione, Mitch Brown, and Donald Sacco. The first draft of the manuscript was written by Alicia Macchione and all authors commented on previous versions of the manuscript. All authors read and approved the final manuscript.

Funding The authors did not receive support from any organization for the submitted work.

Availability of Data/Materials For all study materials and data: https:// osf.io/mxqe4/?view_only=7f3ca7ebf00b4d67a2c8117ee849a5ee.

Code Availability All code for data cleaning and analysis associated with the current submission is available at https://osf.io/mxqe4/?view_ only $=7 \mathrm{f} 3 \mathrm{ca} 7 \mathrm{ebf00b} 4 \mathrm{~d} 67 \mathrm{a} 2 \mathrm{c} 8117 \mathrm{ee} 849 \mathrm{a} 5 \mathrm{ee}$.

\section{Declarations}

Ethics Approval All procedures performed in studies involving human participants were in accordance with the ethical standards of the institutional and/or national research committee and with the 1964 Helsinki Declaration and its later amendments or comparable ethical standards.

Consent to Participate Informed consent was obtained from all individual participants included in the study.
Consent for Publication The authors affirm that human research participants provided informed consent for the publication of this manuscript.

Conflicts of Interests/Competing Interests All authors certify that they have no affiliations with or involvement in any organization or entity with any financial interest or non-financial interest in the subject matter or materials discussed in this manuscript.

\section{References}

Alper, S., Bayrak, F., \& Yilmaz, O. (2021). All the Dark Triad and some of the Big Five traits are visible in the face. Personality and Individual Differences, 168, 110350.

Baum, A., \& Valins, S. (1979). Architectural mediation of residential density and control: Crowding and the regulation of social contact. Advances in Experimental Social Psychology, 12, 131-175.

Bertl, B., Bertl, B., Pietschnig, J., Tran, U. S., Stieger, S., Stieger, S., \& Voracek, M. (2017). More or less than the sum of its parts? Mapping the Dark Triad of personality onto a single Dark Core. Personality and Individual Differences, 114, 140-144.

Borras-Guevara, M. L., Batres, C., \& Perrett, D. I. (2017). Domestic violence shapes Colombian women's partner choices. Behavioral Ecology and Sociobiology, 71, Article 175.

Brown, M. (2021). Goal relevance and desirability of virtuous behavior in satisfying affiliative and pathogen avoidance needs. Personality and Individual Differences, 181, 111025.

Brown, M., \& Sacco, D. F. (in press). How and when crowd salience activates pathogen-avoidant motives. Evolutionary Behavioral Sciences.

Brown, M., \& Sacco, D. F. (2017). Unrestricted sociosexuality predicts preferences for extraverted male faces. Personality and Individual Differences, 108, 123-127.

Brown, M., \& Sacco, D. F. (2016). Avoiding extraverts: Pathogen concern downregulates preferences for extraverted faces. Evolutionary Psychological Science, 2.

Brown, M., Sacco, D. F., Lolley, K. P., \& Block, D. (2017). Facing the implications: Dangerous world beliefs differentially predict men and women's aversion to facially communicated psychopathy. Personality and Individual Differences, 116, 1-5.

Brown, M., Tracy, R. E., Young, S. G., \& Sacco, D. F. (in press). Crowd salience heightens tolerance to healthy facial features. Adaptive Human Behavior and Physiology.

Campbell, W. K., Bush, C. P., Brunell, A. B., \& Shelton, J. (2005). Understanding the social costs of narcissism: the case of the tragedy of the commons. Personality \& Social Psychology Bulletin, 31(10), 1358-1368. https://doi.org/10.1177/0146167205274855

Debruine, L. (2020). Composite images. https://debruine.github.io/ post/composite-images/

Elleman, L., Condon, D., Holtzman, N., Allen, V., \& Revelle, W. (2020). Smaller is better: Associations between personality and demographics are improved by examining narrower traits and regions. Collabra: Psychology, 6.

Faul, F., Erdfelder, E., Lang, A. G., \& Buchner, A. (2007). G*Power 3: A flexible statistical power analysis program for the social, behavioral, and biomedical sciences. Behavior Research Methods, 39, 175-191.

Glenn, A. L., Kurzban, R., \& Raine, A. (2011). Evolutionary theory and psychopathy. Aggression and Violent Behavior, 16, 371-380.

Gochman, I. R., \& Keating, J. P. (1980). Misattributions to crowding: Blaming crowding for nondensity-caused events. Journal of Nonverbal Behavior, 4, 157-175. 
Gul, P., Kupfer, T., Elmas, P., Kose, E. A., Koskun, T., \& Keesmekers, N. (2021). Disease avoidance motives trade-off against social motives, especially mate-seeking, to predict social distancing: Evidence from the Covid-19 pandemic. Preprint from PsyArXiv.

Holtzman, N. S. (2011). Facing a psychopath: Detecting the dark triad from emotionally-neutral faces, using prototypes from the Personality Faceaurus. Journal of Research in Personality, 45, 648-654.

Holtzman, N. S., \& Strube, M. J. (2012). People with dark personalities tend to create a physically attractive veneer. Social Psychological and Personality Science, 4(4), 461-467.

Jaeger, B., Jones, A., Satchell, L., Schild, C., \& Van Leeuwen, F. (2021). Who likes extraverts? Re-examining motivational tradeoff effects in social perception. Preprint from PsyArXiv.

Jones, A. L., \& Jaeger, B. (2019). Biological bases of beauty revisited: The effect of symmetry, averageness, and sexual dimorphism on female facial attractiveness. Symmetry, 11, 279.

Jones, K. E., Patel, N. G., Levy, M. A., Storeygard, A., Balk, D., Gittleman, J. L., \& Daszak, P. (2008). Global trends in emerging infectious diseases. Nature, 451, 990-993.

Jones, D. N., \& Paulhus, D. L. (2011). The role of impulsivity in the Dark Triad of personality. Personality and Individual Differences, 51(5), 679-682.

Lakens, D., Scheel, A. M., \& Isager, P. M. (2018). Equivalence testing for psychological research: A tutorial. Advances in Methods and Practices in Psychological Science, 259-269.

Little, A. C., \& Perrett, D. I. (2007). Using composite images to assess accuracy in personality attribution to faces. British Journal of Psychology, 98, 111-126.

Lyons, M., \& Simeonov, L. (2016). The undesirable Dark Triad? Women dislike Dark Triad male faces across different mating context and socio-ecological conditions. Personality and Individual Differences, 90, 338-341.

Maeng, A., \& Tanner, R. J. (2013). Construing in a crowd: The effects of social crowding on mental construal. Journal of Experimental Social Psychology, 49, 1084-1088.

Maeng, A., Tanner, R. J., \& Soman, D. (2013). Conservative when crowded: Social crowding and consumer choice. Journal of Marketing Research, 50, 739-752.

Marcinkowska, U. M., Helle, S., \& Lyons, M. T. (2015). Dark traits: Sometimes hot, and sometimes not? Female preferences for Dark Triad faces depend on sociosexuality and contraceptive use. Personality and Individual Differences, 86, 369-373.

Medlin, M. M., Sacco, D. F., \& Brown, M. (2020). The relation between narcissistic personality traits and accurate identification of, and preference for, facially communicated narcissism. Evolutionary Psychological Science, 6, 166-173.

Møller, A. P., Dufva, R., \& Allander, K. (1993). Parasites and the evolution of host social behavior. Advances in the Study of Behavior, $22,65-102$.

Montoya, R. M., \& Insko, C. A. (2008). Toward a more complete understanding of the reciprocity of liking effect. European Journal of Social Psychology, 38, 477-498.

Neuberg, S. L., Kenrick, D. T., \& Schaller, M. (2011). Human threat management systems: Self-protection and disease avoidance. $\mathrm{Neu}$ roscience and Biobehavioral Reviews, 35(4), 1042-1051.
Parkinson, B. (2005). Do facial movements express emotions or communicate motives? Personality and Social Psychology Review, 9, 278-311.

Paulhus, D. L. (1998). Interpersonal and intrapsychic adaptiveness of trait self-enhancement: A mixed blessing? Journal of Personality and Social Psychology, 74(5), 1197-1208. https://doi.org/10. 1037/0022-3514.74.5.1197

Paulhus, D. L., \& Williams, K. M. (2002). The Dark Triad of personality: Narcissism, Machiavellianism and psychopathy. Journal of Research in Personality, 36(6), 556-563.

Rogoza, Radosław \& Cieciuch, Jan. (2020). Dark Triad traits and their structure: An empirical approach. Current Psychology, 39.

Sacco, D. F., \& Brown, M. (2018). The face of personality: Adaptive inferences from facial cues are moderated by perceiver personality and motives. Social and Personality Psychology Compass, 12, e12410.

Sacco, D., Lustgraaf, C., Brown, M., \& Young, S. (2015). Activation of self-protection threat increases women's preferences for dominance in male faces. Human Ethology Bulletin, 30.

Schaeffer, G. H., \& Patterson, M. L. (1980). Intimacy, arousal, and small group crowding. Journal of Personality and Social Psychology, 38, 283-290.

Sell, A., Hone, L. S., \& Pound, N. (2012). The importance of physical strength to human males. Human Nature (Hawthorne, N.Y.), 23(1), 30-44. https://doi.org/10.1007/s12110-012-9131-2

Shiramizu, V. K. M., Kozma, L., DeBruine, L. M., \& Jones, B. C. (2019). Are dark triad cues really visible in faces? Personality and Individual Differences, 139, 214-216.

Sng, O., Neuberg, S. L., Varnum, M. E. W., \& Kenrick, D. T. (2017). The crowded life is a slow life: Population density and life history strategy. Journal of Personality and Social Psychology, 112, 736-754.

Snyder, J. K., Fessler, D. M. T., Tiokhin, L., Frederick, D. A., Lee, S. W., \& Navarrete, C. D. (2011). Trade-offs in a dangerous world: Women's fear of crime predicts preferences for aggressive and formidable mates. Evolution and Human Behavior, 32, 127-137.

Solomon, E., \& Lyons, M. (2020). Not my protector-Women have an aversion to high dark triad faces irrespective of childhood or current environmental danger. Evolutionary Psychological Science, 6, 241-245.

Stockdale, J. E. (1978). Crowding: Determinants and effects. Advances in Experimental Social Psychology, 11, 197-247.

Twenge, J. M., \& Campbell, W. K. (2003). Isn't it fun to get the respect that we're going to deserve?. Narcissism, social rejection, and aggression. Personality and Social Psychology Bulletin, 29, 261-272.

United Nations Department of Economic and Social Affairs. (2017). World Population Prospects 2017.

von Borell, C., Kordsmeyer, T. L., Gerlach, T. M., \& Penke, L. (2019). An integrative study of facultative personality calibration. Evolution and Human Behavior, 40, 235-248.

Publisher's Note Springer Nature remains neutral with regard to jurisdictional claims in published maps and institutional affiliations. 